\title{
Estimativas de Componentes de (Co)variância e Herdabilidade Direta e Materna de Pesos Corporais em Ovinos da Raça Santa Inês ${ }^{1}$
}

\author{
Wandrick Hauss de Sousa², Carmen Silva Pereira ${ }^{3}$, José Aurélio Garcia Bergmann³, \\ Francisco Luiz Ribeiro da Silva ${ }^{4}$
}

\begin{abstract}
RESUMO - O objetivo deste trabalho foi estimar os componentes de (co)variância e herdabilidade direta e materna de pesos corporais em ovinos da raça Santa Inês. Um total de 3971 registros de pesos de crias da raça Santa Inês, filhas de 114 reprodutores e 758 ovelhas, foi analisado, obtido de rebanhos experimentais do SNPA da Embrapa, no período de 1983 a 1995. Componentes de covariância e parâmetros genéticos resultantes de efeito genético aditivo direto, efeitos genético materno e de ambiente permanente, bem como a covariância entre esses efeitos, para peso ao nascer (PN), aos 112 (P112) e 196 dias de idade (P196), foram estimados pelo método da máxima verossimilhança restrita (REML), usando o aplicativo MTDFREML sob modelo animal. Incluindo ou não efeitos genéticos materno ou de ambiente materno permanente, bem como a covariância genética entre os efeitos direto e materno, cinco diferentes modelos de análises foram usados para determinar o modelo mais apropriado para descrever cada uma das características. Em geral, o modelo mais completo (modelo 4 ) foi o que apresentou maior valor para logaritmo da função de verossimilhança ( $\log$ de L) para todas características estudadas. Quando efeitos maternos foram excluidos, estimativas de herdabilidade direta foram substancialmente inflacionadas $(0,31$ a 0,37$)$. Efeitos maternos foram importantes para todas as características, mesmo as medidas após o desmame. Correlações genéticas entre os efeitos direto e materno para PN e P112 foram negativas e de baixa magnitude (-0,31 a - 0,10), porém, para P196, estes valores foram positivos e de alta magnitude $(0,67$ a 0,98$)$. A exclusão dos efeitos maternos, quando importantes, resultou em superestimativa das herdabilidade.
\end{abstract}

Palavras-chave: característica de crescimento, efeito materno, modelo animal, ovino deslanado, parâmetro genético

\section{Estimates of (Co) Variance Components and Direct and Maternal Heritability for Growth Traits in Santa Inês Sheep Breed}

\begin{abstract}
The objective of this work was to estimate the (co) variance components and direct and maternal heritability of the body weights of sheep of Santa Inês breed. A total of 3,771 lambs weights records of the Santa Inês hair sheep breed, progenies of 114 sires, and 758 ewes were analyzed, obtained from experimental herds of EMBRAPA - SNPA from 1985 to 1995 . The covariance components and genetic parameters from additive genetic effects, maternal and permanent environmental effects, as well as the covariance between those effects for birth weight (BW), weight at 112 (W112) and weight at 196 days of age (W196), were estimated by Restricted Maximum Likelihood (REML) using MTDFREML program, under animal model. Including or not the maternal genetic effect or permanent environmental effect, as well as the genetic covariance between the direct and maternal effects, five different animal models were used to determine the most appropriate model for each trait. In general, the most comprehensive model (Model 4) was the one that presented the greatest value for the maximum $\log$ likelihood function ( $\log$ of $\mathrm{L})$ for all studied traits. When maternal effects were excluded, estimates of direct heritabilities were substantially inflated (.31 to .37). The maternal effects were important for all traits, even those that were measured after weaning. Genetic correlation between direct and maternal effects for BW and W112 were negative and low (from -.31 to -.10 ), however, for W196 this value was positive and high (from .67 to .98). The exclusion of the maternal effects, when they are important, resulted in overestimates of heritability.
\end{abstract}

Key Words: growth traits, maternal effect, animal model, hair sheep, genetic parameter

\section{Introdução}

Características de crescimento, como peso ao nascer e ao desmame, mensuradas na fase inicial do desenvolvimento do animal, são importantes na determinação da eficiência econômica de qualquer sistema de produção de ovinos. Algumas destas características podem ser recomendadas como critérios de seleção (OLSON et al., 1976; BADENHORST et al., 1991). Assim, se essas características forem incluídas em programas de melhoramento, estimativas precisas de valores genéticos são necessárias para otimizar os programas de seleção.

\footnotetext{
${ }^{1}$ Parte da Tese de Doutorado apresentada pelo primeiro autor à UFMG.

2 Pesquisador III da EMEPA-PB, Caixa postal 275, CEP 58013-290, João Pessoa - PB.

3 Professor da Escola de Veterinária da UFMG, Minas Gerais.

4 Pesquisador da EMBRAPAICNPC - SOBRAL.
} 
A importância dos efeitos maternos sobre o crescimento, na maioria das espécies domésticas, tem determinado a realização de alguns estudos (ROBISON, 1981; WILLHAN, 1972). Os efeitos maternos são influências pré e pós-natal, principalmente nutricional, da ovelha sobre as crias e surgem da capacidade da mãe em produzir leite e outros comportamentos maternos. Parte do efeito materno pode ser genético e parte, ambiental (ROBINSON, 1996). Em programas de seleção, para maximizar o ganho genético de características influenciadas pelos efeitos maternos, é necessário obter informações não somente dos valores genéticos diretos, mas também dos efeitos maternos. A disponibilidade de modernos programas estatísticos tem facilitado o fracionamento dos componentes de variância e, conseqüentemente, a obtenção desses efeitos, bem como das correlações genéticas e de ambiente entre eles.

Para obtenção do BLUP de valores genéticos, utilizando-se as equações dos modelos mistos, esses componentes devem ser conhecidos, no mínimo, proporcionalmente. Adicionalmente, é importante ressaltar que esses componentes podem variar de acordo com a raça, o ambiente e o sistema de produção utilizado.

Embora a literatura mostre que efeitos maternos em características de crescimento são mais substanciais em ovinos que em bovinos e suínos (BRADFORD, 1972), poucos estudos sobre efeitos maternos para peso e crescimento de crias têm sido feitos, principalmente, em raças deslanadas (MARIA et al., 1993; VAN WYK et al., 1993; TOSH e KEMP, 1994; e AL- SHOREPY e NOTTER, 1996).

Se os efeitos genéticos maternos são também importantes, mas não são considerados nas análises, as estimativas de herdabilidade podem estar viciadas e a eficiência da seleção pode ser reduzida. Isto poderia resultar da correlação negativa entre o efeitos direto e materno, o que resultaria em baixa herdabilidade total (SNYMAN, et al., 1995). Adicionalmente, se a correlação entre o efeito direto e materno for acentuadamente negativa, esperar-se-á grande redução na eficiência do teste de progênie (HANRAHAN 1976, ROBISON, 1981).

Alguns autores (ROBINSON, 1996; LEE e POLLAK, 1997) têm chamado a atenção, principalmente em dados de campo, para as consequências das estimativas de correlação genética entre o efeito direto e materno, quando outros efeitos são ignorados no modelo. Assim, em estudo de dados simulados, ROBINSON (1996) avaliou o efeito da não-inclusão da interação reprodutor $\mathrm{x}$ ano no modelo. $\mathrm{O}$ autor concluiu que, nas análises de dados que apresentam interação reprodutor $x$ ano, a exclusão desse efeito do modelo resultou em correlação negativa entre o efeito direto e materno. LEE e POLLAK (1997), seguindo a mesma estratégia de ROBINSON (1996) e analisando dados de bovinos da raça Simental, concluíram que o efeito desta interação foi significativo, porém com origem desconhecida, e a estimativa da correlação entre os efeitos direto e materno, ignorando a interação touro $\mathrm{x}$ ano, foi negativa $(-0,29)$. Quando consideradas no modelo a interação e a covariância entre efeitos genético direto e materno, a correlação foi de $-0,14$.

Estimativas de correlações genéticas entre o efeito direto e o materno em ovinos, principalmente deslanados, são ainda pouco conhecidas. MARIA et al. (1993) estudaram essas correlações em características de crescimento (pesos pré-desmama) em ovinos da raça Romanov, encontrando estimativas variando de $-0,99$ a 0,99 . Os autores atribuíram esses altos valores ao pequeno tamanho da amostra. SOUSA et al. (1995) estimaram correlações genéticas negativas entre o efeito direto e o materno, para características de crescimento em caprinos da raça Anglonubiana, que variaram de $-0,88$ a - 0,12 , demonstrando que efeitos maternos em caprinos parecem ter o mesmo comportamento.

Os objetivos deste estudo foram determinar os efeitos aleatórios que conduzem a um modelo animal mais eficiente a ser aplicado em características de crescimento na pré e pós-desmama de ovinos da raça Santa Inês e estimar componentes de covariância e parâmetros genéticos para cada uma das características.

\section{Material e Métodos}

Para realização deste estudo foram utilizados registros de 3846 crias da raça Santa Inês, nascidas no período de 1983 a 1995, oriundos de três rebanhos experimentais pertencentes à Empresa Estadual de Pesquisa Agropecuária da Paraíba S.A (EMEPAPB), EMBRAPA - Centro Nacional de Pesquisa de Caprinos (CNPC) e EMBRAPA Centro de Pesquisas Agropecuária dos Tabuleiros Costeiros (CPATC). Características da raça

A raça Santa Inês - encontrada em toda a região Nordeste do Brasil - originou-se do cruzamento da raça Bergamácia, de origem italiana, com ovelhas crioulas e Morada Nova, seguido de um período de seleção e/ou evolução para ausência de lã. É uma 
1254 Rev. bras. zootec.

raça de grande porte, com alto potencial para crescimento e produção de leite, mas com baixa taxa de partos múltiplos (FIGUEIREDO et al., 1983). O padrão da pelagem inclui o branco, o vermelho, o preto e o malhado. Em condições de pastejo, o peso de uma ovelha adulta varia de 40 a $50 \mathrm{~kg}$ e os machos podem atingir até $90 \mathrm{~kg}$. A raça é encontrada, geralmente, em áreas com melhores condições de pastagens e/ou de manejo que as outras raças deslanadas. Descrições mais detalhadas podem ser encontradas em FIGUEIREDO (1986), RAJAD (1987) e SOUSA (1987).

\section{Práticas de manejo e seleção dos rebanhos}

Durante o período de coletas dos dados, as práticas de manejo dos rebanhos foram constantes. Os rebanhos permaneceram em campos de pastagem nativa e nativa melhorada constituidas de capim Buffel (Cenchrus. ciliaris,) e Pangola (Digitária decumbis) durante a maior parte do ano. Nos períodos de escassez de alimentos, os animais receberam no cocho suplementação alimentar à base de silagem, palma forrageira (Opuntia ficus sp) e capim elefante (Peninsetum purpureum) e uma ração concentrada, quando necessário. A suplementação mineral foi prática constante durante todo o ano. O plano sanitário consistia de vacinações contra febre aftosa, controle sistemático de endo e ecto-parasitas e tratamento clínico da linfadenite caseosa.

Após a edição dos dados, foram excluídos os registros correspondentes às seguintes restrições: (i) reprodutores com menos de quatro progênies; (ii) registros de pesos de crias natimortas; (iii) registros incompletos; e (iv) crias nascidas de partos triplos $(0,03 \%)$, que foram considerados como duplos.

Após as restrições nos dados, foi considerado um total de 3791 registros de pesos ao nascer, 3293 ao desmame (90-112 dias de idade) e 2568 aos 196 dias de idade. Progênies de 114 reprodutores e 758 ovelhas foram usadas nas análises.

Na Tabela 1 estão apresentadas a distribuição do número de observações e algumas estatísticas descritivas para as características de crescimento, de acordo com rebanho, estação, sexo da cria, idade da ovelha e tipo de nascimento e criação.

Os pesos corporais das crias foram ajustados para idades padrão de 112 e 196 dias, por meio de interpolações, utilizando-se as pesagens mensais mais próximas dessas idades, com base na seguinte fórmula: (ex: peso ao desmame).

$\mathrm{PD}=\mathrm{PD}_{\mathrm{i}}+\left[\left(\mathrm{PD}_{\mathrm{i}}-\mathrm{P} 84\right) /(\mathrm{IDA}-\mathrm{ID} 84) *(112-\mathrm{IDA})\right]$ em que

$\mathrm{PD}=$ peso da cria ao desmame, ajustado para 112 dias de idade;

$\mathrm{PD}_{\mathrm{i}}=$ peso da cria na data do desmame;

P84 = peso da cria aos 84 dias de idade;

IDA = idade da cria no desmame; e

ID84 = idade da cria na pesagem aos 84 dias.

Análises preliminares foram conduzidas pelo método dos quadrados mínimos, por intermédio do procedimento GLM do SAS (1989), para identificar os efeitos não-genéticos que poderiam influenciar os pesos das crias ao nascer (PN), aos 112 (P112) e 196 dias de idade (P196). Após processadas as análises iniciais, os efeitos fixos considerados nos modelos foram: grupo contemporâneo, rebanho-ano-estação, sexo da cria, efeito combinado do tipo de nascimentocriação (TNC), idade da ovelha e a covariável peso da ovelha ao parto, conforme apresentadas na Tabela 2. Para peso ao nascer, o efeito do tipo de nascimento foi utilizado ao invés da combinação tipo de nascimento-criação. As classes da combinação (TNC) foram tipo de nascimento simples, criado como simples (11), tipo de nascimento duplo, criado como duplo (22), e tipo de nascimento duplo, criado como simples (21). A classe tipo de nascimento-criação foi estabelecida mediante o seguinte critério: crias nascidas de partos duplos que sobreviveram até 56 dias de idade tinham seu tipo de nascimento mantido; caso contrário, era mudado.

Os componentes de variância e covariância, bem como os parâmetros genéticos, foram estimados por meio do método da Máxima Verossimilhança Restrita - REML, utilizando-se um algoritmo livre de derivadas DFREML (GRASER et al., 1987), com o aplicativo MTDFREML (BOLDMAN et al., 1995). Modelos animais univariados foram ajustados para todas as características. Foram propostos cinco diferentes modelos de análises, por meio da inclusão ou exclusão de efeitos genéticos maternos ou de ambiente materno permanente.

$$
\begin{aligned}
& Y=X \beta+Z_{1} a+e \\
& Y=X \beta+Z_{1} a+Z_{2} m+e \\
& \text { Assumindo a cov }(a, m)=A \sigma_{a m} \\
& Y=X \beta+Z_{1} a+Z_{3} p e+e \\
& Y=X \beta+Z_{1} a+Z_{2} m+Z_{3} p e+e \\
& \text { Assumindo } a \operatorname{cov}(a, m)=A \sigma_{a m} \\
& Y=X \beta+Z_{1} a+Z_{2} m+Z_{3} p e+e \\
& \text { Assumindo a cov }(a, m)=0
\end{aligned}
$$

em que Y é um vetor ( $\mathrm{N}$ x 1) de observação do animal; $\beta$, o vetor de efeitos fixos no modelo, associados com a matriz de incidência $\mathrm{X}$; a, o vetor dos 
SOUSA et al.

efeitos genéticos diretos, associado com a matriz de incidência $Z_{1} ; \mathrm{m}$, o vetor dos efeitos genéticos maternos, associado com a matriz de incidência $Z_{2}$; pe, o vetor dos efeitos de ambiente materno permanentes, associado com a matriz de incidência $\mathrm{Z}_{3}$; e $e$, o vetor de resíduos aleatórios. A é a matriz de coeficientes de parentesco entre os animais; I, uma matriz identidade; e $\sigma_{a m}$, a covariância entre os efeitos genéticos direto e materno. A estrutura básica da matriz de variância e covariância para as análises foi, então, descrita como:

$$
V\left[\begin{array}{c}
a \\
m \\
p e \\
e
\end{array}\right]=\left[\begin{array}{cccc}
A \sigma_{a}^{2} & A \sigma_{a m} & 0 & 0 \\
A \sigma_{a m} & A \sigma_{m}^{2} & 0 & 0 \\
0 & 0 & I \sigma_{p e}^{2} & 0 \\
0 & 0 & 0 & I \sigma_{e}^{2}
\end{array}\right]
$$

em que, para obtenção dos valores iniciais dos componentes de (co)variância, a verossimilhança foi, primeiro, maximizada somente em relação aos componentes de covariância, fixando-se as variâncias. Em seguida, a verossimilhança foi maximizada em relação aos componentes de variância, fixando-se as covariâncias aos valores obtidos na primeira fase. Finalmente, a maximização foi executada em relação a todos os componentes (MEYER, 1994). Cada análise foi reiniciada com diferentes valores iniciais, para garantir que os valores dos componentes estimados correspondessem ao máximo absoluto da função de verossimilhança, na tentativa de evitar a convergência para um máximo local. O critério de convergência foi considerado quando a variância do "Simplex" foi inferior a $10^{-9}$.

Os componentes de variância estimados incluíram: $\sigma_{\mathrm{a}}^{2}$, variância genética aditiva direta; $\sigma^{2}{ }_{\mathrm{m}}$, variância genética aditiva materna; $\sigma_{\mathrm{am}}$, a covariância genética entre os efeitos direto e materno; $\sigma^{2}$, a variância devido aos efeitos permanentes de ambiente da mãe (ovelha); e $\sigma_{\mathrm{e}}^{2}$, a variância residual. A partir dos componentes estimados, pode-se derivar os seguintes parâmetros: $\mathrm{h}^{2}{ }_{\mathrm{a}}=\sigma_{\mathrm{a}}^{2} / \sigma^{2}{ }_{\mathrm{P}}$ herdabilidade direta; $\mathrm{h}_{\mathrm{m}}^{2}=\sigma_{\mathrm{m}}^{2} / \sigma_{\mathrm{P}}^{2}$ herdabilidade materna; $\mathrm{r}_{\mathrm{Gam}}$, correlação genética entre os efeitos direto e materno; $c^{2}$, razão de variância do ambiente permanente para a variância fenotípica $\sigma_{\mathrm{P}}^{2}$; a herdabilidade total dos efeitos genéticos aditivos totais $\mathrm{h}_{\mathrm{T}}^{2}=\left(\sigma_{\mathrm{a}}^{2}+0,5 \sigma^{2} \mathrm{~m}+1,5 \sigma_{\mathrm{a}, \mathrm{m}}\right), \sigma_{\mathrm{p}}^{2}$, significando a regressão do genótipo total de um animal (direto e materno) sobre seu fenótipo (WILLHAM, 1972); e $\mathrm{C}_{\mathrm{a}, \mathrm{m}=} \sigma_{\mathrm{am}} / \sigma_{\mathrm{p}}^{2}$, covariância genética entre efeitos direto e materno como proporção da variância fenotípica.

Então, por esse modelo, a variância fenotípica foi estimada: em que

$$
\sigma_{P}^{2}=\left(\sigma_{a}^{2}+3 / 2 \sigma_{a m}+1 / 2 \sigma_{m}^{2}+\sigma_{e}^{2}\right)
$$

$\sigma^{2}{ }_{a}=$ variância genética aditiva;

$\sigma_{\mathrm{m}}^{2}=$ variância materna;

$\sigma^{2}{ }_{\text {am }}=$ covariância entre o efeito genético direto e materno; e

$\sigma_{\mathrm{e}}^{2}=$ variância do erro.

\section{Teste da razão de verossimilhança}

A determinação da superioridade de um modelo sobre o outro foi feita por meio de comparações da razão de verossimilhança de modelos seqüencialmente reduzidos (RAO, 1973). A razão de verossimilhança (LR) foi calculada para testar a significância de um modelo $\mathrm{M}_{\mathrm{i}}$, contendo um ou mais parâmetros adicionais, comparado com o modelo $\mathrm{M}_{\mathrm{j}}$, porém com a omissão daqueles parâmetros. Seu valor é -2 log LR (menos duas vezes o logaritmo natural da razão de verossimilhança, definida como $\mathrm{L}_{\mathrm{j}} / \mathrm{L}_{\mathrm{i}}$, em que $\mathrm{L}_{\mathrm{j}}$ é o máximo da verossimilhança restrita para o modelo $\mathrm{M}_{\mathrm{j}}$ e $\mathrm{L}_{\mathrm{i}}$, o máximo para o modelo $\mathrm{M}_{\mathrm{i}}$.

\section{Resultados e Discussão}

As estimativas dos componentes de variância para peso ao nascimento, peso ao desmame, peso aos 196 dias de idade, bem como a diferença entre (-) menos 2 vezes as funções de verossimilhança de dois diferentes modelos, estão sumarizadas na Tabela 3 . As estimativas de parâmetros genéticos das análises univariadas, bem como outros parâmetros derivados dessas estimativas, são apresentadas na Tabela 4.

\section{Peso ao nascimento}

O peso da cria ao nascimento teve forte influência dos efeitos maternos aditivo e de ambiente permanente (Modelos II, III e IV), contribuindo para melhorar os valores do logarítmo da função de verossimilhança, quando comparado com o valor obtido no modelo I. No entanto, o modelo completo (Modelo IV) foi o que mais contribuiu com a maximização do logaritmo da função de verossimilhança, ou, equivalentemente, com a minimização de -2 log LR. Adicionalmente, o modelo IV considerou ambas as similaridades entre irmãos completos e entre crias nascidas da mesma ovelha em diferentes anos.

Os efeitos maternos foram responsáveis por 17 a $21 \%$ da variância fenotípica, contribuindo com a redução da herdabilidade direta de 0,37 , no modelo I, para 0,12 , no modelo II, e 0,15 , no modelo III. Não houve diferença significativa $(\mathrm{P}>0,01)$ entre os modelos IV e V, evidenciando que a covariância entre os 
Rev. bras. zootec.

Tabela 1- Número de observações e algumas estatísticas descritivas para as características de crescimento, de acordo com rebanho, estação, sexo da cria, idade da ovelha, tipo de nascimento e de criação

Table 1 - Number of records and some descriptive statistics for growth traits, according to herd-year, sex of the lamb, age of ewe and type of birth and rearing classes

\begin{tabular}{|c|c|c|c|}
\hline \multirow[t]{2}{*}{$\begin{array}{l}\text { Parâmetro } \\
\text { Parameter }\end{array}$} & \multirow[t]{2}{*}{$\begin{array}{c}\text { Peso ao nascer } \\
\text { Birth weight }\end{array}$} & \multicolumn{2}{|c|}{$\begin{array}{c}\text { Peso } \\
\text { Weight }\end{array}$} \\
\hline & & $112 \mathrm{~d}$ & $196 \mathrm{~d}$ \\
\hline № observações & 3791 & 3293 & 2568 \\
\hline \multicolumn{4}{|l|}{ № of records } \\
\hline Média & 3,58 & 19,17 & 22,57 \\
\hline \multicolumn{4}{|l|}{ Mean } \\
\hline Desvio-padrão & 0,77 & 4,88 & 5,05 \\
\hline \multicolumn{4}{|l|}{ Standard deviation } \\
\hline $\mathrm{CV} \%$ & 21,5 & 25,4 & 22,4 \\
\hline \multicolumn{4}{|l|}{ Classe } \\
\hline \multicolumn{4}{|l|}{ Class } \\
\hline \multicolumn{4}{|l|}{ Idade (anos) } \\
\hline \multicolumn{4}{|l|}{ Age (years) } \\
\hline$<2$ & 863 & 779 & 665 \\
\hline $2-3$ & 736 & 656 & 523 \\
\hline $3-4$ & 687 & 610 & 478 \\
\hline $4-5$ & 580 & 500 & 373 \\
\hline $5-6$ & 425 & 354 & 254 \\
\hline $6-7$ & 309 & 248 & 193 \\
\hline$>7$ & 191 & 147 & 82 \\
\hline \multicolumn{4}{|l|}{ Sexo da cria } \\
\hline \multicolumn{4}{|l|}{ Sex of the lamb } \\
\hline Macho & 1953 & 1693 & 1299 \\
\hline \multicolumn{4}{|l|}{ Male } \\
\hline Fêmea & 1838 & 1600 & 1269 \\
\hline \multicolumn{4}{|l|}{ Female } \\
\hline \multicolumn{4}{|l|}{ Tipo de nascimento } \\
\hline \multicolumn{4}{|l|}{ Type of birth } \\
\hline Simples & - & 1966 & 1510 \\
\hline \multicolumn{4}{|l|}{ Single } \\
\hline Duplos & - & 1327 & 1058 \\
\hline \multicolumn{4}{|l|}{ Multiple } \\
\hline \multicolumn{4}{|l|}{ Tipo nascimento- criação } \\
\hline \multicolumn{4}{|l|}{ Type of birth-rearing } \\
\hline $1-1$ & 2146 & 1966 & 1510 \\
\hline $2-2$ & 352 & 979 & 810 \\
\hline $2-1$ & 1293 & 348 & 248 \\
\hline \multicolumn{4}{|l|}{ Rebanho } \\
\hline \multicolumn{4}{|l|}{ Herd } \\
\hline 1 & 1027 & 864 & 820 \\
\hline 2 & 1951 & 1794 & 1748 \\
\hline 3 & 813 & 635 & - \\
\hline \multicolumn{4}{|l|}{ Estação de nascimento } \\
\hline Lambing season & & & \\
\hline Chuvosa (março/julho) & 3385 & 2967 & 2453 \\
\hline Rainy (March/July) & & & \\
\hline Seca (agosto/fevereiro) & 406 & 326 & 115 \\
\hline Dry (August/February) & & & \\
\hline
\end{tabular}

efeitos direto e materno, a princípio, pode ser negligenciada. Os resultados obtidos neste estudo estão de acordo com os relatados por SNYMAN et al. (1995) e AL-SHOREPY e NOTTER (1996), os quais também verificaram forte influência do efeito materno sobre o peso ao nascimento das crias.
Estimativas de herdabilidade direta obtidas neste estudo para peso ao nascer estão dentro do intervalo daquelas normalmente relatadas na literatura, por vários autores, para diferentes raças e métodos. Com base nos resultados obtidos no modelo II para peso ao nascer, fica evidente que a estimativa da herdabilidade materna $\left(\mathrm{h}^{2}{ }_{\mathrm{m}}\right)$ foi superestimada, já que o efeito de ambiente permanente materno não foi incluído no modelo, o que foi comprovado pelo modelo IV. A variância materna, nesse caso, também incluiu a variância, devido ao efeito permanente de ambiente da ovelha, mostrando a dificuldade de se decompor o efeito materno. Isso poderia explicar a herdabilidade materna $\left(\mathrm{h}_{\mathrm{m}}^{2}\right)$ relativamente alta relatada por VAN WYK et al. (1993), os quais não incluíram no modelo o efeito materno de ambiente permanente.

O valor obtido para o componente da ovelha de 0,0948 (Tabela 3), para peso ao nascer, está próximo de 0,10 obtido por MARIA et al. (1993), porém é menor que os compreendidos entre 0,27 e 0,37 relatados por TOSH e KEMP (1994) e maior que 0,02 estimado por SWAN e HICKSON (1994), os quais utilizaram modelo idêntico ao adotado neste estudo. Normalmente, esses valores de componente da ovelha obtidos para peso ao nascer têm sido atribuídos aos efeitos de ambiente permanente do ambiente uterino, bem como aos efeitos de nascimentos múltiplos.

As estimativas de covariância entre o efeito direto e materno foram negativas e próximas de zero (-0,01 e -0,008). Estes resultados estão próximos dos obtidos por VAN WYK et al. (1993) e NASHOLM e DANELL (1996), que relataram, respectivamente, estimativas de $-0,05$ e 0,01 , porém estão muito distantes das obtidas por MARIA et al. (1993) e TOSH e KEMP (1994), que obtiveram estimativas negativas e próximas da unidade.

Estimativa de correlação genética entre os efeitos direto e materno para esta característica foi negativa e de pequena magnitude $(-0,15)$, próxima à obtida por TOSH e KEMP (1994) para a raça Ramanov $(-0,13)$, mas muito diferente dos valores de -0,99 e 0,37 obtidos por MARIA et al. (1993) e TOSH e KEMP (1994), respectivamente; e de 0,10 e $-0,56$ obtidos por NASHOLM e DANELL (1996), para diferentes raças. Causas dessas correlações negativas têm sido apontadas na literatura como possível covariância ambiental negativa entre mãe e filho, que não é considerada (BAKER, 1990; MEYER, 1992; e SWALWE, 1993). ROBINSON (1996) considera que as estimativas negativas são, provavelmente, consequência de variação adicional entre reprodutores ou da variação da interação reprodutor $x$ ano. 
Tabela 2 - Sumário da estrutura dos dados e efeitos fixos incluídos no modelo para cada característica analisada

Table 2 - Summary of data structure and fixed effects included in the model for each trait analyzed

\begin{tabular}{|c|c|c|c|}
\hline \multirow[t]{2}{*}{$\begin{array}{l}\text { Estrutura dos dados } \\
\text { Data structure }\end{array}$} & \multirow[t]{2}{*}{$\begin{array}{l}\text { Peso ao nascer } \\
\text { Birth weight }\end{array}$} & \multicolumn{2}{|c|}{$\begin{array}{c}\text { Peso } \\
\text { Weight }\end{array}$} \\
\hline & & $112 d$ & $196 \mathrm{~d}$ \\
\hline Animais com registro & & & \\
\hline $\begin{array}{l}\text { Progeny with records } \\
\text { Animais no pedigree }\end{array}$ & 3791 & 3293 & 2568 \\
\hline $\begin{array}{l}\text { Animals in pedigree } \\
\text { Reprodutores }\end{array}$ & 4439 & 4439 & 4439 \\
\hline $\begin{array}{l}\text { Sire } \\
\text { Ovelhas }\end{array}$ & 114 & 105 & 88 \\
\hline $\begin{array}{l}\text { Ewes } \\
\text { Progênie por reprodutor }\end{array}$ & 758 & 686 & 448 \\
\hline $\begin{array}{l}\text { Progeny per sire } \\
\text { Efeitos fixos }\end{array}$ & 33,2 & 31,3 & 29,1 \\
\hline Fixed effects fitted & & & \\
\hline $\begin{array}{l}\text { Rebanho-ano-estação } \\
\text { Herd-year-season }\end{array}$ & $\checkmark$ & $\checkmark$ & $\boldsymbol{V}$ \\
\hline $\begin{array}{l}\text { Tipo de nascimento } \\
\text { Type of birth }\end{array}$ & $\checkmark$ & $\checkmark$ & $\checkmark$ \\
\hline $\begin{array}{l}\text { Tipo de nasc.-criação } \\
\text { Type birth-rearing class }\end{array}$ & - & $\checkmark$ & $\checkmark$ \\
\hline $\begin{array}{l}\text { Sexo da cria } \\
\text { Sex of lamb }\end{array}$ & $\checkmark$ & $\checkmark$ & $\checkmark$ \\
\hline $\begin{array}{l}\text { Idade da ovelha } \\
\text { Age of ewe }\end{array}$ & $\checkmark$ & $\checkmark$ & $\checkmark$ \\
\hline $\begin{array}{l}\text { Peso da ovelha ao parto } \\
\text { Ewe weight at lambing }\end{array}$ & $\checkmark$ & $\checkmark$ & $\checkmark$ \\
\hline
\end{tabular}

As estimativas de herdabilidades total $\left(\mathrm{h}^{2}{ }_{\mathrm{T}}\right)$ obtidas para peso ao nascer, variando de 0,16 a 0,19 , estão muito próximas às obtidas por TOSH e KEMP (1994), que relataram valores de 0,11 a 0,25.

\section{Peso à desmama}

Quando, opcionalmente, foi incluído no modelo o efeito materno aditivo ou permanente de ambiente (Modelos II e III), houve aumento significativo no valor do logaritmo da função de verossimilhança ( $\log \mathrm{L})$ sobre o valor obtido no modelo mais simples (Modelo I). Quando os efeitos maternos foram ignorados (Modelo I), obtiveram-se estimativas, para $\sigma_{a}^{2} \mathrm{e}$ $\mathrm{h}^{2} \mathrm{a}$, substancialmente maiores que para os outros modelos. A inclusão do efeito materno aditivo (Modelo II) resultou em substancial aumento no valor do $\log$ L sobre o valor obtido no Modelo I, demostrando a existência de forte efeito materno sobre o peso à desmama. A inclusão desse efeito no modelo contribuiu com $25 \%$ da variância total sobre o peso à desmama e reduziu as estimativas de $\sigma^{2}{ }_{\mathrm{a}}$ e $\sigma_{\mathrm{e}}^{2}$.

Quando foi considerado o efeito permanente de ambiente, em vez do efeito materno aditivo, também houve considerável aumento no valor do $\log \mathrm{L}$ (sobre o Modelo I), evidenciando a importância deste efeito sobre o peso à desmama. Isto resultou em redução de $83 \%$ na estimativa da herdabilidade direta $\left(\mathrm{h}^{2}{ }_{\mathrm{a}}\right)$, comparada àquela obtida no modelo I. Entretanto, com base no valor do log L, os dados foram melhor ajustados, quando o modelo incluiu os efeitos materno aditivo e permanente de ambiente (Modelo IV). Estes efeitos contribuíram, respectivamente, apenas com 10,4 e $13,8 \%$ da variância fenotípica, reduzindo a herdabilidade direta $\left(\mathrm{h}^{2}{ }_{\mathrm{a}}\right)$ da característica de 0,37 (Modelo I) para 0,04 (Modelo IV). Nas Tabelas 3 e 4 são apresentados dados de como os efeitos direto e permanente podem estar confundidos na decomposição da variância materna. Visto que no modelo II (exclusão do efeito permanente), $\sigma^{2}$ pe foi de 2,5458; no modelo III (exclusão do efeito materno aditivo), $\sigma_{\mathrm{m}}^{2}=2,9252$; e no modelo IV (inclusão dos dois efeitos), $\sigma_{\mathrm{m}}^{2}=1,1736$ e $\sigma_{\mathrm{pe}}^{2}=1,5605$, deve-se ter bastante cautela no estudo destes componentes.

A estimativa de herdabilidade $\left(\mathrm{h}_{\mathrm{m}}^{2}\right)$ (Modelo IV) para peso aos 112 dias de idade foi baixa, sendo próxima à relatada por NASHOLM e DANELL(1996), que obtiveram estimativas $\left(\mathrm{h}^{2} \mathrm{~m}\right)$, para peso aos 120 dias de idade, de 0,11. Ao contrário, AL-SHOREPY e NOTTER (1996), utilizando o mesmo modelo deste estudo, obtiveram estimativas para peso, aos 90 e 120 dias idade, de 0,08 e 0,0, respectivamente.

$\mathrm{O}$ valor de $\mathrm{c}^{2}=0,14$ obtido para peso aos 112 dias (desmama), pelo modelo IV, está muito próximo de 0,16 ; 0,19; e 0,12, para pesos aos 120 e 100 dias de idade, respectivamente, reportados por TOSH e KEMP (1994) e AL-SHOREPY e NOTTER (1996). Os valores obtidos para $\mathrm{c}^{2}$ (Modelos II e IV), considerando o erropadrão, não apresentaram diferença significativa.

Estimativas de covariância entre os efeitos direto e maternos de -0,35 e -0,067, obtidas para esta característica (Modelos II e IV), também foram negativas, mas diferentes entre si. Estimativas negativas, mas com maior magnitude $(-0,60)$ para peso aos 90 dias de idade, foi relatada por VAN WYK et al. (1993). Ao contrário, NASHOLM e DANELL (1996) obtiveram estimativa positiva $(0,78)$ de covariância para peso aos 120 dias. Este fato mostra, também, o possível problema na decomposição da variância materna, visto que no modelo II não foi incluído o efeito permanente e, no modelo IV, foram incluídos os dois efeitos.

Estimativas de correlação genética entre os efeitos direto e materno para peso aos 112 dias de idade, também, foram negativas $(-0,31$ e $-0,10)$ como as 
Rev. bras. zootec.

Tabela 3 - Estimativas de componentes de variância $\left(\mathrm{kg}^{2}\right)$ e diferença entre a função do logaritmo da verossimilhança de dois diferentes modelos animal, para pesos das crias ao nascer, aos 112 e aos 196 dias de idade

Table 3 - (Co)variance components estimates $\left(\mathrm{kg}^{2}\right)$ and the difference between likelihood functions of two different animal models for weights of the lamb at birth (BW), 112 (W112) and 196 days of age (W196)

\begin{tabular}{|c|c|c|c|c|}
\hline \multirow[t]{2}{*}{$\begin{array}{l}\text { Modelo } \\
\text { Model }\end{array}$} & \multirow[t]{2}{*}{$\begin{array}{l}\text { Parâmetro } \\
\text { Parameter }\end{array}$} & \multirow[t]{2}{*}{$\begin{array}{c}\text { Peso ao nascer } \\
\text { Birth weight }\end{array}$} & \multicolumn{2}{|c|}{$\begin{array}{c}\text { Peso } \\
\text { Weight }\end{array}$} \\
\hline & & & $112 \mathrm{~d}$ & $196 d$ \\
\hline I & $\begin{array}{l}\sigma_{\mathrm{a}_{2}^{2}}^{2} \\
\sigma_{\mathrm{e}}^{2} \\
\sigma_{\mathrm{p}}^{2}\end{array}$ & $\begin{array}{l}0,1753 \\
0,2953 \\
0,4707\end{array}$ & $\begin{array}{c}4,4383 \\
7,5527 \\
11,9910\end{array}$ & $\begin{array}{c}4,9297 \\
10,8467 \\
15,7764\end{array}$ \\
\hline$\left(M_{1}-M_{2}\right)$ & $\begin{array}{c}\sigma_{\mathrm{a}}^{2} \\
\sigma_{\mathrm{m}}^{2} \\
\sigma_{\mathrm{am}} \\
\sigma_{\mathrm{e}_{2}}^{2} \\
\sigma_{\mathrm{p}}^{2} \\
-\mathbf{2} \log \mathbf{L} \mathbf{R}^{\mathbf{a}}\end{array}$ & $\begin{array}{c}0,0553 \\
0,0948 \\
-0,010 \\
0,3149 \\
0,4542 \\
93,32 * *\end{array}$ & $\begin{array}{c}0,4707 \\
2,9252 \\
-0,358 \\
8,4243 \\
11,4618 \\
91,35 * *\end{array}$ & $\begin{array}{c}0,8923 \\
1,5913 \\
0,796 \\
11,9901 \\
15,2700 \\
47,40 * *\end{array}$ \\
\hline$\left(M_{1}-M_{3}\right)$ & $\begin{array}{c}\sigma_{\mathrm{a}}^{2} \\
\sigma_{\mathrm{pe}} \\
\sigma_{\mathrm{e}_{2}} \\
\sigma_{\mathrm{p}}^{2} \\
\mathbf{- 2} \log \mathbf{L R}\end{array}$ & $\begin{array}{c}0,0667 \\
0,0767 \\
0,3046 \\
0,4480 \\
88,20 * * \\
\end{array}$ & $\begin{array}{c}0,6502 \\
2,5458 \\
8,0698 \\
11,2659 \\
97,45 * *\end{array}$ & $\begin{array}{c}1,5028 \\
2,2579 \\
11,3462 \\
15,1070 \\
34,79 * *\end{array}$ \\
\hline $\begin{array}{l}\left(M_{2}-M_{4}\right) \\
\left(M_{3}-M_{4}\right) \\
\end{array}$ & $\begin{array}{c}\sigma_{\mathrm{a}}^{2} \\
\sigma_{\mathrm{m}}{ }^{2} \\
\sigma_{\mathrm{am}} \\
\sigma_{\mathrm{pe}} \\
\sigma_{\mathrm{e}} \\
\sigma_{\mathrm{p}}{ }^{2} \\
-2 \log \mathbf{L R} \\
-2 \log \mathbf{L R}\end{array}$ & $\begin{array}{c}0,0569 \\
0,0541 \\
-0,008 \\
0,0375 \\
0,3086 \\
0,4490 \\
7,960 * * \\
12,98 * * \\
\end{array}$ & $\begin{array}{c}0,4202 \\
1,1736 \\
-0,067 \\
1,5605 \\
8,1949 \\
11,2815 \\
15,72 * * \\
9,62 * *\end{array}$ & $\begin{array}{c}0,8409 \\
0,5930 \\
0,690 \\
1,2829 \\
11,7149 \\
15,1225 \\
-4,46 \\
8,15 * *\end{array}$ \\
\hline$\left(M_{4}-M_{5}\right)$ & $\begin{array}{c}\sigma_{\mathrm{a}}^{2} \\
\sigma_{\mathrm{m}} \\
\sigma_{\mathrm{pe}} \\
\sigma_{\mathrm{e}} \\
\sigma_{\mathrm{p}}^{2} \\
-2 \log \mathbf{L R}\end{array}$ & $\begin{array}{c}0,0519 \\
0,0478 \\
0,0373 \\
0,3116 \\
0,4488 \\
-0,32\end{array}$ & $\begin{array}{c}0,3945 \\
1,1017 \\
1,5679 \\
8,2115 \\
11,2810 \\
-0,03\end{array}$ & $\begin{array}{c}1,0237 \\
1,1655 \\
1,3190 \\
11,6026 \\
15,1111 \\
7,88 * *\end{array}$ \\
\hline
\end{tabular}

** $(P<0,01) ; \sigma^{2}$, variância genética aditiva; $\sigma^{2}{ }_{m}$, variância genética materna; $\sigma^{2}$ pe , variância ambiental materna; $\sigma_{p}^{2}$, variância fenotípica; $\sigma_{e}^{2}$, variância residual.

a $L R=$ razão de verossimilhança definida como $L_{j} / L_{i}$, em que $L_{j}$ é o máximo da verossimilhaça restrita para o modelo $\mathrm{Mj}$ e $\mathrm{L}_{\mathrm{i}}$ e o máximo para o modelo $\mathrm{M}_{\mathrm{i}}$.

${ }^{* *}(\mathrm{P}<01) \sigma^{2}{ }_{a}$, direct additive genetic variance; $\sigma^{2}{ }_{m}$, maternal additive genetic variance; $\sigma^{2}{ }_{p e}$, maternal environmental variance; $\sigma_{p}{ }^{2}$ phenotypic variance; $\sigma_{e}^{2}$ residual variance.

a $L R=$ likelihood ratio expressed as $L_{j} L_{j}$, where $L_{j}$ is the maximum of the restricted likelihood for $M_{j}$ model and $L_{i}$ is the maximum for $M_{i}$ model.

obtidas para peso ao nascer. Resultados similares foram relatados por VAN WYK et al. (1993) e TOSH e KEMP (1994), porém foram diferentes das obtidas por NASHOLM e DANELL (1996), que obtiveram estimativas altas e positivas.

Estimativas dessa correlação em dados de campo são freqüentemente mais negativas que as obtidas com dados experimentais (NASHOLM e DANELL, 1996). Além disso, muitos autores têm discutido a possibilidade de que a covariância ambiental negativa entre mãe e filho, que não é considerada, vicia para baixo a correlação genética entre os efeitos genéticos direto e maternos (BAKER, 1990; MEYER, 1992; e SWALWE, 1993).
As estimativas de herdabilidades total $\left(\mathrm{h}^{2}{ }_{\mathrm{T}}\right)$ encontradas para esta característica foram muito baixas, diferentes daquelas obtidas por VAN WYK et al. (1993), TOSH e KEMP (1994) e NASHOLM e DANELL (1996), que relataram estimativas de $\left(\mathrm{h}^{2}{ }_{\mathrm{T}}\right)$ para pesos aos 100-120 dias de idade, variando de 0,18 a 0,28 .

Peso aos 196 dias de idade

Os efeitos maternos permaneceram ainda evidentes após a desmama, porém foram menos importantes para peso aos 196 dias de idade. A tendência de declínio desses efeitos era esperada, pois efeitos de ambientes associados com a cria decrescem em 
importância, quando a cria se torna independente da ovelha. Resultados similares foram relatados por MARIA et al. (1993) e TOSH e KEMP (1994). Isto não é surpresa, considerando que o peso aos 196 dias carrega parte do genótipo do efeito materno da ovelha, que pode persistir no período pós-desmama, semelhante ao que ocorre com os efeitos do ambiente materno, idade da ovelha e tipo de nascimento (OLSON et al., 1976; ATKINS, 1986). Também pode ter ocorrido estresse sobre a cria durante a fase da pré-desmama, devido à baixa produção de leite da ovelha, refletindo no genótipo materno, o que pode resultar em crescimento compensatório após o desmame, como o que acontece, por exemplo, quando o nível de produção de leite de uma ovelha de primeira ordem de partoé limitado, ou quando ela pare duas ou mais crias (CH'ANG e RAE, 1970). O modelo IV foi significativamente melhor que o modelo III, mas não inferior ao modelo II, indicando que o efeito materno total foi difícil de ser fracionado.
Estimativas de herdabilidades direta $\left(\mathrm{h}^{2}{ }_{\mathrm{a}}\right)$ e materna $\left(\mathrm{h}^{2}{ }_{\mathrm{m}}\right)$ obtidas para esta característica variaram de baixa a moderadas, evidenciando que a herdabilidade materna não deve ser negligenciada às idades próximas à desmama. As estimativas obtidas nos modelos (II e IV) para peso aos 196 dias de idade foram ainda bastantes significativas, indicando que o efeito de ambiente permanente devido à ovelha (mãe) foi tão importante quanto o genótipo do animal, na determinação do peso aos 196 dias de idade.

Quando não foi considerada a covariância entre o efeito direto e materno $\left(\sigma_{\mathrm{am}}\right)$ no modelo completo (Modelo V), houve significativo aumento no valor do $\log \mathrm{L}$, evidenciando, assim, a não necessidade de considerar covariância, no modelo, para essa característica. Ao contrário do que ocorreu com os pesos ao nascer e aos 112 dias de idade, as estimativas de covariância e correlação genética obtidas, nesta pesquisa, entre os efeitos direto e materno (Modelos II e IV) foram altas e positivas, com valores que variaram

Tabela 4 - Estimativas de parâmetros genéticos para pesos das crias ao nascer, aos 112 e 196 dias de idade

Table 4 - Estimates of genetics parameters for weights of the lambs at birth (BW), at 112 (W112) and 196 days of age (W196)

\begin{tabular}{|c|c|c|c|c|}
\hline \multirow[t]{2}{*}{$\begin{array}{l}\text { Modelo } \\
\text { Model }\end{array}$} & \multirow[t]{2}{*}{$\begin{array}{l}\text { Parâmetro } \\
\text { Parameter }\end{array}$} & \multirow[t]{2}{*}{$\begin{array}{c}\text { Peso ao nascer } \\
\text { Birth weight }\end{array}$} & \multicolumn{2}{|c|}{$\begin{array}{c}\text { Peso } \\
\text { Weight }\end{array}$} \\
\hline & & & $112 d$ & 196d \\
\hline I & $\begin{array}{l}\mathrm{h}^{2} \\
\mathbf{h}^{\mathbf{2}^{\mathrm{a}}}\end{array}$ & $\begin{array}{l}0,37 \pm 0,04 \\
0,37 \pm 0,04\end{array}$ & $\begin{array}{l}0,37 \pm 0,04 \\
0,37 \pm 0,04\end{array}$ & $\begin{array}{l}0,31 \pm 0,04 \\
0,31 \pm 0,04\end{array}$ \\
\hline II & $\mathrm{h}_{\mathrm{a}}^{2}$ & $0,12 \pm 0,04$ & $0,04 \pm 0,04$ & $0,06 \pm 0,03$ \\
\hline & $\begin{array}{c}\mathrm{h}_{\mathrm{m}}^{2} \\
\mathbf{r}_{\mathbf{G a m}} \\
\mathrm{C}_{\mathrm{am}} \\
\mathbf{h}_{\mathbf{T}}^{\mathbf{2}_{\mathbf{T}}} \\
\end{array}$ & $\begin{array}{c}0,21 \pm 0,04 \\
-0,15 \pm 0,23 \\
-0,022 \\
0,19\end{array}$ & $\begin{array}{c}0,26 \pm 0,04 \\
-0,31 \pm 0,21 \\
-0,003 \\
0,12\end{array}$ & $\begin{array}{c}0,10 \pm 0,02 \\
0,67 \pm 0,31 \\
0,005 \\
0,19\end{array}$ \\
\hline III & $\begin{array}{l}\mathrm{h}^{2} \\
\mathrm{c}^{2^{\mathrm{a}}} \\
\mathrm{h}^{2} \mathrm{~T}\end{array}$ & $\begin{array}{l}0,15 \pm 0,04 \\
0,17 \pm 0,02 \\
0,15 \pm 0,04\end{array}$ & $\begin{array}{l}0,06 \pm 0,02 \\
0,23 \pm 0,02 \\
0,06 \pm 0,02\end{array}$ & $\begin{array}{l}0,10 \pm 0,03 \\
0,15 \pm 0,02 \\
0,10 \pm 0,03\end{array}$ \\
\hline IV & $\begin{array}{c}\mathrm{h}^{2} \\
\mathrm{~h}^{2} \mathrm{~m} \\
\mathbf{C}_{\mathbf{a m}} \\
\mathbf{r}_{\mathbf{G a m}} \\
\mathbf{c}^{2} \\
\mathbf{h}_{\mathbf{T}}^{\mathbf{2}}\end{array}$ & $\begin{array}{c}0,13 \pm 0,04 \\
0,12 \pm 0,04 \\
-0,020 \\
-0,15 \pm 0,13 \\
0,084 \pm 0,03 \\
0,16\end{array}$ & $\begin{array}{c}0,04 \pm 0,02 \\
0,10 \pm 0,04 \\
-0,006 \\
-0,10 \pm 0,47 \\
0,14 \pm 0,04 \\
0,08\end{array}$ & $\begin{array}{c}0,06 \pm 0,03 \\
0,04 \pm 0,02 \\
0,004 \\
0,98 \pm 0,55 \\
0,085 \pm 0,03 \\
0,08\end{array}$ \\
\hline $\mathbf{V}$ & $\begin{array}{l}\mathrm{h}^{2} \\
\mathrm{~h}^{2} \mathrm{a} \\
\mathbf{c}^{2} \\
\mathbf{h}^{2}\end{array}$ & $\begin{array}{c}0,12 \pm 0,03 \\
0,11 \pm 0,04 \\
0,083 \pm 0,03 \\
0,16\end{array}$ & $\begin{array}{c}0,03 \pm 0,02 \\
0,10 \pm 0,03 \\
0,14 \pm 0,03 \\
0,08\end{array}$ & $\begin{array}{c}0,07 \pm 0,03 \\
0,08 \pm 0,03 \\
0,087 \pm 0,03 \\
0,10\end{array}$ \\
\hline
\end{tabular}

$\mathbf{h}^{2}{ }_{\mathbf{a}}$ é a herdabilidade direta; $\mathbf{h}^{2}{ }_{\mathrm{m}}$, a herdabilidade materna; $\mathbf{C}_{\mathrm{am}}=\sigma_{\mathrm{am}} / \sigma^{2}{ }_{\mathbf{p}}$, covariância entre efeitos direto e materno como proporção da variância fenotípica ; $\sigma_{\mathrm{am}}$, covariâncla genética entre os efeitos direto e maternos; $r_{G a m}$, correlação genética entre os efeitos direto e materno; $c^{2}=\sigma^{2}{ }_{\mathbf{p e}} / \sigma^{2}{ }_{\mathbf{p}} ; \mathbf{h}^{2}{ }_{\mathrm{T}}$, herdabilidade total - $\mathrm{h}^{2} \mathrm{~T}=\left(\sigma^{2}{ }_{\mathrm{a}}+1,5 \sigma_{\mathrm{am}}+0,5 \sigma^{2}{ }_{\mathrm{a}}\right) / \sigma^{2}{ }_{\mathrm{p}}$

$\boldsymbol{h}^{2}{ }_{a}$ is direct heritability; $\boldsymbol{h}^{2}{ }_{m}$, maternal heritability; $\boldsymbol{C}_{a m}=\sigma_{a m} / \sigma^{2}{ }_{p}$, covariance between direct and maternal effects as a proportion of the phenotypic variance; $\sigma_{a m}$ direct and maternal genetic covariance; $\boldsymbol{r}_{\text {Gam }}$, direct and maternal genetic correlation; $\boldsymbol{c}^{2}=\sigma^{2}{ }_{p e} / \sigma^{2}{ }_{p} ; \boldsymbol{h}^{2}{ }_{T}$, total heritability $h^{2}{ }_{T}=\left(\sigma^{2}{ }_{a}+1,5 \sigma_{a m}+0,5 \sigma^{2}{ }_{a}\right) / \sigma^{2}{ }_{p}$. 
de 0,69 a 0,79 e 0,67 a 0,98 , respectivamente, para as covariâncias e correlações genéticas. Nenhum estudo considerando esse aspecto (covariância e correlação) com ovinos nestas idades, ou próximas, foi encontrado na literatura.

As estimativas da herdabilidade total $\left(\mathrm{h}^{2}{ }_{\mathrm{T}}\right)$, obtidas para peso aos 196 dias de idade, apresentaram comportamento similar às obtidas para as outras características, variando de 0,08 a 0,19 , sendo maior nos modelos mais simples. O modelo mais simples (Modelo I) superestimou a variância genética aditiva e o potencial para resposta à seleção, principalmente, porque ignorou a correlação entre os efeitos genéticos direto e materno.

A literatura tem relatado tendência de a herdabilidade direta aumentar com a idade do animal (MARIA et al., 1993; TOSH e KEMP, 1994; e NASHOLM e DANELL, 1996), o que também pode ser observado nos resultados obtidos neste estudo (Modelo IV), no entanto, os resultados encontrados por GJEDREN (1967) e RICORDEAU (1990) não revelaram essa tendência.

Em ovinos, como também em bovinos, resultados de correlações genéticas entre os efeitos diretos e maternos são de algum modo contraditórios. MEYER (1992), utilizando dados de bovinos, obteve estimativas para essa correlação ora positivas, ora negativas, dependendo da raça. MARIA et al. (1993) obtiveram estimativas de correlação genéticas altas e negativas entre esses efeitos, para diversos pesos em ovinos. ROBISON (1972) verificou essa correlação negativa entre os efeitos direto e materno pode ser induzida pelo ambiente. MEYER (1992) e SWALWE (1993) também concluíram que essa correlação poderia ser amplificada pelo sistema de manejo. NASHOLM e DANELL (1996) relataram que estimativas dessa correlação, utilizando dados de campo, tendem a ser mais negativas que aquelas com dados experimentais.

\section{Análises para duas características}

A realização de análises pareadas entre as características foram feitas utilizando o modelo II, em vez do modelo IV, para reduzir o número de componentes de covariâncias a serem estimados, uma vez que este modelo incluiu os efeitos genético aditivo direto e materno, bem como apropriadas covariâncias entre as características.

Constam da Tabela 5 estimativas de correlações genéticas e fenotípicas entre os pesos estudados. As correlações genéticas foram baixas, sendo negativas entre pesos ao nascimento e aos 196 dias de idade $(-0,11)$. As correlações fenotípicas foram todas positivas e variaram de 0,16 a 0,95 , sendo a mais alta aquela entre peso ao nascer e aos 112 dias de idade $(0,95)$. As correlações genéticas obtidas foram bastante diferentes daquelas relatadas por AL-SHOREPY e NOTTER (1996) e MARIA et al. (1993). Estimativas de correlações genéticas negativas, entre peso ao nascer e aos 365 dias e entre peso aos 112 - 365 dias de idade de $-0,39$ e -0,63, respectivamente, em ovinos deslanados da raça Morada Nova, foram também relatadas por OLIVEIRA (1992).

Tabela 5 - Médias e amplitudes das estimativas de herdabilidades $^{1}$ e estimativas de correlações ${ }^{2}$ genética e fenotípica entre os pesos ao nascer (PN), aos 112 (P112) e 196 dias de idade (P196)

Table 5 - Means and range of estimates of heritabilities and genetic and phenotypic correlation among weights at birth (BW), at 112 (W112) and 196 days of age (W196)

\begin{tabular}{lccc}
\hline Característica & $\begin{array}{c}\text { Peso ao nascer } \\
\text { Trait }\end{array}$ & \multicolumn{2}{c}{$\begin{array}{c}\text { Peso } \\
\text { Beight }\end{array}$} \\
\cline { 3 - 4 } & & 0,37 & $-0,11$ \\
PN & $\mathbf{0 , 0 9}$ & & \\
BW & $(0,05-0,13)$ & $\mathbf{0 , 0 5}$ & 0,28 \\
P112 & 0,95 & $(0,03-0,07)^{3}$ & \\
W112 & & 0,18 & $\mathbf{0 , 0 8}$ \\
P196 & 0,16 & & $(0,05-0,11)$ \\
W196 & &
\end{tabular}

${ }^{1}$ Herdabilidade e a média das estimativas obtidas nas diversas análises, pareada (na diagonal).

2 Correlações genética e fenotípica estão acima e abaixo da diagonal, respectivamente.

${ }^{1}$ Os valores entre patênteses representam a variação das herdabilidades nas análises pareadas.

1 Means and heritability estimates obtained in several analyses, from two traits (in the diagonal).

2 Genetic and phenotypic correlation are above and below the main diagonal, respectively.

3 Values between brackets represent the variation of the heritabilities in both traits analyses. 


\section{Conclusões}

Ao ignorar os efeitos maternos diretos, que na realidade existem, pode-se chegar à superestimação das herdabilidades direta e total. Do mesmo modo, quando se ignora o efeito permanente de ambiente materno, a análise resulta em estimativa de herdabilidade materna superestimada.

Efeitos maternos apresentaram influência importante em todas as características estudadas, mesmo após a desmama.

Os diversos modelos mostraram possível confundimento entre os diversos componentes do efeito materno, indicando cautela no desdobramento desses efeitos.

O modelo completo (Modelo IV) foi o mais apropriado na estimativa de herdabilidades de características de crescimento, quando efeitos maternos são importantes.

As estimativas de correlações genéticas positivas significativas entre os efeitos direto e materno, obtidas para peso aos 196 dias de idade, mostram que a seleção para aumentar esse peso nas crias irá também melhorar a capacidade materna da ovelha.

A importância relativa dos efeitos materno e direto varia com a idade.

A ovelha (mãe) teve maior influência na prédesmama.

\section{Referências Bibliográficas}

AL-SHOREPY, S., NOTTER, D.R. 1996. Genetic variation and covariation for ewe reproduction, lamb growth, and lamb scrotal circunference in a fall-lambing sheep flock. J. Anim. Sci., 74:1490-1498.

ATKINS, K.D. 1986. A genetic analysis of components of lifetime productivity in Scottish Blackface sheep. Anim. Prod., 43:405-419.

BAKER, R.L. 1990. The role of maternal effects on the efficiency of selection in beef cattle: a review. Proc. N. Z. Soc. Anim. Prod., 40:285-303.

BADENHORST, M.A., OLIVIER, J.J., SCOEMAN, S.J. et al. 1991. Ondersoek na seleksiemaatsatawwe by Afrinoskape. Genetic parameters in Afrinos sheep. S. Afr. J. Anim. Sci., 21(3):162-165.

BRADFORD, G.E. 1972. The role of maternal effects in animal breeding: VII. Maternal effects in sheep. J. Anim. Sci., 35:1324-1337.

BOLDMAN, K.G., KRIESE, L.A., VAN VLECK, L.D. et al. 1995. A manual for use of MTDFREML; a set of programs to obtain estimates os variances and covariances [DRAFT]. Lincoln: Department of Agriculture. 120p.

CH'ANG, T.S., RAE, A.L. 1970. The genetic basis of growth, reproduction and maternal environment in Romney ewes. I. Genetic variation in gogget charaters and fertility of the ewe. Aust. J. Agric. Res., 21:115-123.

FIGUEIREDO, E.A.P., OLIVEIRA, E.R., BELLAVER, C. et al. 1983. Hair sheep performance in Brazil. In: FITZHUGH, H. A., BRADFORD, G. E. (Eds.). Hair sheep of Western Africa and the Americas. Colorado: Westview Press, Boulder. p.125-140.

FIGUEIREDO, E.A.P. Potential breeding plans developed from observed genetic parameters and simulated genotypes for Morada Nova sheep in Northeast Brazil. Texas: Texas A\&M University, 1986. 196p. Tese (Doutorado em Ciencia Animal) - Texas A\&M University, 1986.

GJEDREN, T. 1967. Phenotypic and genetic parameters for weight of lambs at five ages. Acta Agr. Scand., 17:359-365.

GRASER, H.U., SMITH, S.P., TIER, B. 1987. A derivative-free approach for estimating variance components in animal models by restricted maximum likelihood. J. Anim. Sci., 64:1362.

HANRAHAN, J. P. 1976. Maternal effects and selection response with na application to sheep data. Anim. Prod., 22:359-366.

LEE, C., POLLAK, E. J. 1997. Relationship between sire x year interactions and direct-maternal genetic correlation for weaning weight of simmental cattle. J. Anim. Sci., 75:68-75.

MARIA, G.A., BOLDMAN, K.G., VAN VLECK, L.D. 1993. Estimates of variance due to direct and maternal effects for growth traits of Romanov sheep. J. Anim. Sci., 71:845-852.

MEYER, K. Variance components due to direct and maternal effects for growth traits of Australian beef cattle. Livest. Prod. Sci., 31:179-184, 1992.

MEYER, K. 1994. Estimates of direct and maternal correlations among growth traits in Australian beef cattle. Livest. Prod. Sci., 38:91-96.

NÄSHOLM, A., DANELL, Ö. 1996. Genetic relationships of lamb weigth, maternal ability, and mature ewe weight in swedish finewool sheep. J. Anim. Sci., 74:329-339.

OLIVEIRA, S.M.P. Desempenho de ovinos da raça Morada Nova variedade branca no estado do Ceará: Parâmetros genéticos $e$ de ambiente. Belo Horizonte: UFMG; Tese (Doutorado) Escola de Veterinária, 1992. 69p.

OLSON, L.W., DICKERSON, G.E., CROUSE, J.D et al. 1976. Selection criteria for intensive market lamb production: carcass na growth traits. J. Anim. Sci., 43:90-97.

RAJAB, M.H. Simulation of genetic and environmental interactions of three tropical gair sheep breeds for meat production. Texas: Texas A\&M University, College Station, 1987. 155p. Tese (Doutorado em Ciência Animal) - Texas A\&M University, 1987.

RAO, C.R. Minimum variance quadratic unbiased estimation of variance components. J. Mult. Anal., 1:445-452, 1973.

RICORDEAU, G., THIMONIER, J., POIVEI, J.P. et al. 1990. Research on the Romanov Sheep Breed in France: A review. Livest. Prod. Sci., 24:305-312.

ROBISON, O.W. 1972. The role of maternal effects in animal breeding: V. Maternal effects in swine. J. Anim. Sci., 35:13031311.

ROBISON, O.W. 1981. The influence of maternal effects on the efficiency of selection; a review. Livest. Prod. Sci., 8:121-137.

ROBINSON, D.L. 1996. Estimation and interpretation of direct and maternal genetic parameters for weights of Australian Angus cattle. Livest. Prod. Sci., 45:1-11.

SAS INSTITUTE. 1989. SAS. user's guide: statistics. SAS Institute Inc., Cary, NC.

SNYMAN, M.A., ERASMUS, G.J., VAN WIK, J.B. 1995. Non genetic factors influencing growth and fleece trais in Afrino sheep. S. Afr. J. Anim. Sci., 25:55-62.

SOUSA, W.H. Genetic and environmental factors affecting growth and reproductive performance of Santa Ines sheep en the semearid region of Brazil. Texas: Texas A\&M University, 1987. 
1262 Rev. bras. zootec.

79p. Dissertação (Mestrado em Zootecnia) - Texas A\&M University, 1987.

SOUSA, W.H. TORRES, R.A., LEITE, P.R.M. Estimativas de componentes de variância devido aos efeitos direto e materno para características de crescimento em caprinos In: REUNIÃO ANUAL DA SOCIEDADE BRASILEIRA DE ZOOTECNIA, 32, 1995. Brasilia, DF. Anais... Brasília, DF: SBZ, 1995. p.721

SWALWE, H.H. 1993. Estimation of direct and maternal (co)variance compnents for growth traits in Australian Simmental beef cattle. J. Anim. Sci., 110:241-248.

SWAN, A.A., HICKSON, J. D. 1994. Maternal effects in Australian Merinos. Proc. 5th World Congr. Gen. Appl. Livest. Prod., 18:143-146.

TOSH, J.J., KEMP, R.A. 1994. Estimations of variance components for lamb weights in three sheep populations. $J$. Anim. Sci., 72:1184-1191.
VAN WYK, J.B., VAN DER SCHIFF, W., ERASMUS, G.J. et al. 1993. Variance components and heritability estimates of early growth traits in the Elsenburg Dormer sheep stud. $S$. Afr. J. Anim. Sci., 23(3/4):72-76.

WILLHAM, R.L. 1972. The role of maternal effects in animal breeding: III. Biometrical aspects of maternal effects in animals. J. Anim. Sci., 35:1288-1295.

Recebido em: 27/10/98

Aceito em: 29/04/99 